\title{
Fixed point theorems for a class of maps in normed Boolean vector spaces
}

Swaminath Mishra', Rajendra Pant ${ }^{1 *}$ and Venkat Murali ${ }^{2}$

* Correspondence: pant. rajendra@gmail.com

'Department of Mathematics,

Walter Sisulu University, Mthatha 5117, South Africa

Full list of author information is available at the end of the article

\section{Abstract}

In this article, we obtain some fixed and common fixed point theorems for a class of maps on normed Boolean vector spaces satisfying the property (E.A) without using continuity. Our results extend and unify some known results.

2000 Mathematics Subject Classification: Primary 06E30.

Keywords: Boolean vector space, Boolean metric, property (E.A), fixed point

\section{Introduction and preliminaries}

Fixed point theory of Boolean functions has many potential applications to errorcorrecting codes, to switching circuits and to the relationship between the consistency of a Boolean equation, cryptography, convergence of some recursive parallel array processes in Boolean arrays and many others. However, there are only a limited number of results available in literature dealing with fixed point theory for Boolean valued functions (see, for instance, Ghilezan [1] and Rudeanu [2]). In addition, most of these results are in finite dimensional spaces. Recently, Rao and Pant [3] obtained some fixed and common fixed point theorems for asymptotically regular maps on finite dimensional normed Boolean vector spaces (for details of Boolean vector spaces, we refer to Subrahmanyam $[4,5])$. The purpose of this article is to obtain some coincidence and common fixed point theorems in infinite dimensional normed Boolean vector spaces for certain classes of maps without using continuity conditions. These maps satisfy the property (E.A) introduced and studied by Aamri and Moutawakil [6] for the first time. It is interesting to note that the above property presents a nice generalization of non-compatible maps. Results obtained herein extend certain results of $[6,7]$ among others to normed Boolean vector spaces.

Definition 1.1. [6] Let $X$ be a metric space and $S, T: X \rightarrow X$. Then the maps $S$ and $T$ are said to satisfy the property (E.A) if there exits a sequence $\left\{x_{n}\right\}$ in $X$ such that

$$
\lim _{n \rightarrow \infty} S x_{n}=\lim _{n \rightarrow \infty} T x_{n}=t, \quad \text { for some } t \in X .
$$

When $T=I$, the identity map on $X$, we obtain the corresponding definition for a single map satisfying the property (E.A) (see [7]).

Example 1.2. [6]. Let $X=[0, \infty)$ endowed with the usual metric. Define $S, T: X \rightarrow X$ by $S x=\frac{x}{4}$ and $T x=\frac{3 x}{4}$ for all $x \in X$. Consider a sequence $x_{n}=\frac{1}{n}$. Clearly $\lim _{n \rightarrow \infty} S x_{n}=\lim _{n \rightarrow \infty} T x_{n}=0$, and $S$ and $T$ satisfy the property (E.A).

(c) 2012 Mishra et al; licensee Springer. This is an Open Access article distributed under the terms of the Creative Commons Attribution License (http://creativecommons.org/licenses/by/2.0), which permits unrestricted use, distribution, and reproduction in any medium, provided the original work is properly cited. 
There are maps which do not satisfy the property (E.A).

Example 1.3. [6]. Let $X=[2, \infty)$ endowed with the usual metric. Define $S, T: X \rightarrow X$ by $S x=x+1$ and $T x=2 x+1$, for all $x \in X$. Suppose $S$ and $T$ satisfy the property (E.A), then there exists a sequence $\left\{x_{n}\right\}$ in $X$ satisfying $\lim _{x \rightarrow \infty} S x_{n}=\lim _{x \rightarrow \infty} T x_{n}=t$, for some $t \in X$. Therefore $\lim _{x \rightarrow \infty} x_{n}=t-1$ and $\lim _{x \rightarrow \infty} x_{n}=\frac{t-1}{2}$. Then $t=1$, which is a contradiction since $1 \notin X$. Hence $S$ and $T$ do not satisfy the property $(E . A)$.

The class of maps satisfying property (E.A) contains the class of the well-known compatible maps (see Jungck [8]) as well as the class of non-compatible maps. The property $(E . A)$ is very useful in the study of fixed points of nonexpansive maps. In fact the property (E.A) ensure the existence of a coincidence point for a pair of nonexpansive type maps in a metric space [7].

For the completeness, we recall the certain definitions and examples from [4].

Definition 1.4. [4]. Let $\mathfrak{V}=(\mathfrak{V},+)$ be an additive abelian group and $\mathscr{B}=\left(\mathscr{B},+, . \prime^{\prime}\right)$ a Boolean algebra. The set $\mathfrak{V}$ with two operations namely 'addition' and 'scalar multiplication' is said to be a Boolean vector space over $\mathscr{B}$ (or simply, a $\mathscr{B}$ - vector space) if for all $x, y \in \mathfrak{V}$ and $a, b \in \mathscr{B}$,

(i) $a(x+y)=a x+a y$;

(ii) $(a b) x=a(b x)=b(a x)$;

(iii) $1 x=x$; and

(iv) if $a b=0$, then $(a+b) x=a x+b x$.

The elements of $\mathfrak{V}$ and $\mathscr{B}$ will be denoted respectively, by $x, y, z$ and $a, b, c$ (with or without indices); the zero of $\mathfrak{V}$ and also null-element of $\mathscr{B}$ will both be denoted by 0 , while the universal element $\left(=0^{\prime}\right)$ of $\mathscr{B}$ will be denoted by 1 .

Example 1.5. [4]. Let $\mathscr{B}$ be any Boolean algebra and $\mathfrak{V}$ be the additive group of the corresponding Boolean ring; then $\mathfrak{V}$ is a $\mathscr{B}$ - vector space if we define: For $a \in \mathscr{B}$ and $x \in \mathfrak{V}, a x=$ the (Boolean) product of $a$ and $x$ in $\mathscr{B}$.

Example 1.6. [4]. Let $R$ be any Ring with unity element 1 and let $\mathscr{B}$ denotes the set of all the central idempotents of $R$; then it is known that $\left(\mathscr{B}, \cup, \cap,^{\prime}\right)$ is a Boolean algebra, where, by definition, $a \cup b=a+b-a b, a \cap b=a b$ and $a^{\prime}=1-a$. If $\mathfrak{V}$ is the additive group of the ring $R$, and for $a \in \mathscr{B}$ and $x \in \mathfrak{V}, a x=$ the product of $a$ and $x$ in $R$, then $\mathfrak{V}$ is a Boolean vector space over $\left(\mathscr{B}, \cup, \cap,^{\prime}\right)$.

Definition 1.7. [4]. A Boolean vector space $\mathfrak{V}$ over a Boolean algebra $\mathscr{B}$ is said to be $\mathscr{B}$-normed (or simply, normed) if and only if there exists a map $\|$.$\| (called norm):$ $\mathfrak{V} \rightarrow \mathscr{B}$ such that

(i) $\|x\|=0$ if and only if $x=0$, and

(ii) $\|a x\|=a\|x\|$ for all $a \in \mathscr{B}$ and $x \in \mathfrak{V}$.

In view of [[4], Corollary 3.2] we note the following.

Let $\mathfrak{V}$ be a $\mathscr{B}$-normed vector space and $\mathfrak{V} \times \mathfrak{V} \rightarrow \mathscr{B}$ then $d(x, y)=\|x-y\|$ defines a Boolean metric on $\mathfrak{V}$, i.e.,

(i) $d(x, y)=0$ if and only if $x=y$;

(ii) $d(x, y)=d(y, x)$ and

(iii) $d(x, z)<d(x, y)+d(y, z)$.

Definition 1.8. [4]. Let $\mathscr{B}$ be a $\sigma$-complete (= countably complete) Boolean algebra. If $\left\{a_{n}\right\}$ is a sequence of elements of $\mathscr{B}$, we define: 
$\lim \inf a_{n}=\cup_{k \geq 1} \cap_{n \geq k} a_{n} ;$ and $\lim \sup a_{n}=\cap_{k \geq 1} \cup_{n \geq k} a_{n} ;$

and if

$$
\liminf a_{n}=a=\lim \sup a_{n}
$$

then we say that $a_{n}$ converges to $a$, and will be written as $a_{n} \rightarrow a$. A sequence $\left\{a_{n}\right\}$ in $\mathscr{B}$ is a Cauchy sequence if and only if $d^{*}\left(a_{n}, a_{m}\right) \rightarrow 0$, where $d^{*}$ is the Boolean metric on $\mathscr{B}$ defined by $d^{*}(a, b)=a^{\prime} b+a b^{\prime}$.

Definition 1.9. [4]. If $\left\{x_{n}\right\}$ is a sequence of elements of $\mathfrak{V}$, we say that $x_{n} \rightarrow x$ $(x \in \mathfrak{V})$ if and only if $\left\|x_{n}-x\right\| \rightarrow 0$; and a sequence $\left\{x_{n}\right\}$ in $\mathfrak{V}$ is Cauchy if and only if $\left\|x_{n}-x_{m}\right\| \rightarrow 0$.

The following definition is the consequence of Definitions 1.1 and 1.4.

Definition 1.10. Let $\mathfrak{V}$ be a normed Boolean vector space and $S, T: \mathfrak{V} \rightarrow \mathfrak{V}$. Then the maps $S$ and $T$ are said to satisfy the property (E.A) if there exits a sequence $\left\{x_{n}\right\}$ in $\mathfrak{V}$ such that

$$
\lim _{n \rightarrow \infty} S x_{n}=\lim _{n \rightarrow \infty} T x_{n}=t, \text { for some } t \in \mathfrak{V} .
$$

\section{Main results}

Let $\mathfrak{V}$ be a normed Boolean vector space and $T: \mathfrak{V} \rightarrow \mathfrak{V}$. A point $z \in \mathfrak{V}$ is called a fixed point of $T$, if $T z=z$. The point $z$ is called a coincidence point of $S, T: \mathfrak{V} \rightarrow \mathfrak{V}$, if $S z=T z$ and a common fixed point, if $z=S z=T z$.

For the sake of brevity, we shall use the following denotations:

$$
\begin{aligned}
& d(x, y):=\|x-y\| \\
& m(x, y):=\max \{d(x, y), d(x, T x), d(y, T y), d(x, T y), d(y, T x)\} ; \\
& M(x, y):=\max \{d(T x, T y), d(T x, S x), d(T y, S y), d(T x, S y), d(T y, S x)\} \\
& g(x, y):=d(x, y)+d(x, S x)+d(y, S y) \\
& G(x, y):=d(S x, S y)+d(S x, T x)+d(S y, T y) .
\end{aligned}
$$

Let $\Phi$ denotes the class of all functions $\psi: \mathscr{B} \rightarrow \mathscr{B}$ satisfying:

(i) $\psi$ is continuous;

(ii) $\psi(a)<a$ '.

Example 2.1. Let $A$ be a non-empty set and $\mathcal{B}$ the class of all subsets of $A$ with three set operation $\cap, \mathrm{U}$, ' (union, intersection, and complement). Then $\mathcal{B}$ defines a Boolean algebra. Now, let $\varphi: \mathcal{B} \rightarrow \mathcal{B}$ be the function defined by

$$
\varphi(a)=a-1 \text { for all } a \in \mathcal{B},
$$

where 1 denotes the universal element of $\mathcal{B}$. Then $\phi \in \Phi$.

Now we obtain a coincidence theorem for a pair of self-maps on a normed Boolean vector space.

Theorem 2.2. Let $\mathfrak{V} b e$ a normed Boolean vector space and $S, T: \mathfrak{V} \rightarrow \mathfrak{V} s u c h$ that

(A) $S \mathfrak{V} \subseteq T \mathfrak{V}$

(B) the maps $S$ and $T$ satisfy the property (E.A);

(C) $d(S x, S y)=\psi(M(x, y))$ for all $x, y \in \mathfrak{V}$, where $\psi \in \Phi$.

If $S \mathfrak{V}$ or TV is a complete subspace of $\mathfrak{V}$ then $S$ and $T$ have a coincidence in $\mathfrak{V}$. 
Further, $S$ and $T$ have a unique common fixed point provided that $S S u=S u$ and $S$ and $T$ commute at the coincidence point.

Proof. Since the maps $S$ and $T$ satisfy the property (E.A) there exits a sequence $\left\{x_{n}\right\}$ in $\mathfrak{V}$ such that

$$
\lim _{n \rightarrow \infty} S x_{n}=\lim _{n \rightarrow \infty} T x_{n}=t, \text { for some } t \in \mathfrak{V} .
$$

Suppose $T \mathfrak{V}$ is a complete subspace of $\mathfrak{V}$ then there exists a point $u \in \mathfrak{V}$ such that $T u=t$.

Using $(C)$, we get

$$
\begin{aligned}
d\left(S x_{n}, S u\right) & =\varphi\left(M\left(x_{n}, u\right)\right) \\
& =\psi\left(\max \left\{d\left(T x_{n}, T u\right), d\left(T x_{n}, S x_{n}\right), d(T u, S u), d\left(T x_{n}, S u\right), d\left(T u, S x_{n}\right)\right\}\right) .
\end{aligned}
$$

Making $n \rightarrow \infty$, we obtain $d(S u, T u)=\psi(d(S u, T u))<(d(S u, T u))^{\prime}$. Which follows that $S u=T u$ and $u$ is a coincidence point of $S$ and $T$.

Further, if $S S u=S u$, and the maps $S$ and $T$ commute at their coincidence point $u$ then $S u=S T u=T S u$ and $S u$ is a common fixed point of $S$ and $T$.

The case in which $S \mathfrak{V}$ is a complete subspace of $\mathfrak{V}$, the condition $S \mathfrak{V} \subseteq T \mathfrak{V}$ implies that there exists a point $u \in \mathfrak{V}$ such that $T u=t$ and the previous proof works.

To prove the uniqueness of common fixed point, we suppose $z_{1}, z_{2}$ are two common fixed points of $S$ and $T$. Then $S z_{1}=T z_{1}=z_{1}$ and $S z_{2}=T z_{2}=z_{2}$. Using the condition (C)

$$
d\left(z_{1}, z_{2}\right)=d\left(S z_{1}, S z_{2}\right)=\psi\left(M\left(z_{1}, z_{2}\right)\right)<\left(d\left(z_{1}, z_{2}\right)\right)^{\prime} .
$$

Which follows that $z_{1}=z_{2}$.

Corollary 2.3. Let $\mathfrak{V}$ be a complete normed Boolean vector space and $S: \mathfrak{V} \rightarrow \mathfrak{V}$ such that

(I) $S$ satisfies the property (E.A)

(II) $d(S x, S y)=\psi(m(x, y))$ for all $x, y \in \mathfrak{V}$, where $\psi \in \Phi$.

Then $S$ has a unique fixed point.

Proof. This comes from Theorem 2.2 when $T$ is an identity map on $\mathfrak{V}$. $\square$

Theorem 2.4. Let $\mathfrak{V} b e$ a normed Boolean vector space and $S, T: \mathfrak{V} \rightarrow \mathfrak{V}$ such that

(A) $S \mathfrak{V} \subseteq T \mathfrak{V}$

(B) the maps $S$ and $T$ satisfy the property (E.A);

(C) $d(S x, S y)=\psi(G(x, y))$ for all $x, y \in \mathfrak{V}$, where $\psi \in \Phi$.

If $S \mathfrak{V}$ or TVV is a complete subspace of $\mathfrak{V}$ then $S$ and $T$ have a coincidence in $\mathfrak{V}$.

Further, $S$ and $T$ have a unique common fixed point provided that $S S u=S u$ and $S$ and $T$ commute at the coincidence point.

Proof. Since the maps $S$ and $T$ satisfy the property (E.A) there exits a sequence $\left\{x_{n}\right\}$ in $\mathfrak{V}$ such that

$$
\lim _{n \rightarrow \infty} S x_{n}=\lim _{n \rightarrow \infty} T x_{n}=t, \text { for some } t \in \mathfrak{V} .
$$

Suppose $T \mathfrak{V}$ is a complete subspace of $\mathfrak{V}$ then there exists a point $u \in \mathfrak{V}$ such that $T u=t$.

Using (C), we get

$$
\begin{aligned}
d\left(S x_{n}, S u\right) & =\psi\left(G\left(x_{n}, u\right)\right) \\
& =\psi\left(d\left(T x_{n}, T u\right)+d\left(T x_{n}, S x_{n}\right)+d(T u, S u)\right) .
\end{aligned}
$$


Making $n \rightarrow \infty$, we obtain $d(S u, T u)=\psi(d(S u, T u))<(d(S u, T u))^{\prime}$. Which follows that $S u=T u$ and $u$ is a coincidence point of $S$ and $T$.

Further, if $S S u=S u$, and the maps $S$ and $T$ commute at their coincidence point $u$ then $S u=S T u=T S u$ and $S u$ is a common fixed point of $S$ and $T$.

The case in which $S \mathfrak{V}$ is a complete subspace of $\mathfrak{V}$, the condition $S \mathfrak{V} \subseteq T \mathfrak{V}$ implies that there exists a point $u \in \mathfrak{V}$ such that $T u=t$ and the previous proof works.

To prove the uniqueness of common fixed point, we suppose $z_{1}, z_{2}$ are two common fixed points of $S$ and $T$. Then $S z_{1}=T z_{1}=z_{1}$ and $S z_{2}=T z_{2}=z_{2}$. Using the condition (C)

$$
d\left(z_{1}, z_{2}\right)=d\left(S z_{1}, S z_{2}\right)=\psi\left(G\left(z_{1}, z_{2}\right)\right)<\left(d\left(z_{1}, z_{2}\right)\right)^{\prime} .
$$

Which follows that $z_{1}=z_{2}$. $\square$

Corollary 2.5. Let $\mathfrak{V}$ be a complete normed Boolean vector space and $S: \mathfrak{V} \rightarrow \mathfrak{V}$ such that

(I) $S$ satisfies the property (E.A);

(II) $d(S x, S y)=\psi(g(x, y))$ for all $x, y \in \mathfrak{V}$, where $\psi \in \Phi$.

Then $S$ has a unique fixed point.

Proof. This comes from Theorem 2.4 when $T$ is an identity map on $\mathfrak{V}$. $\square$

Now we present an example to illustrate our results.

Example 2.6. Let $A$ be a non-empty set and $\mathscr{B}$ the class of all subsets of $A$. Then the class $\mathscr{B}$ with three set operation,$+ \cdot \cdot$, ' (union, intersection, and complement) defines a Boolean algebra. Further, this class $\mathscr{B}$ with the set operation "exclusive-or addition" $\oplus$ (symmetric difference of sets) defines a Boolean ring. Let $\mathfrak{V}=(\mathfrak{V}, \oplus)$ be the additive abelian group of this Boolean ring. For $a$ in $\mathscr{B}$ and $x$ in $\mathfrak{V}$, we define $a x=a \cdot x$ (the Boolean) product of $a$ and $x$ in $\mathscr{B}$. Then $\mathfrak{V}$ is a Boolean vector space over $\mathscr{B}$.

Let $S, T: \mathfrak{V} \rightarrow \mathfrak{V}$ be self-maps defined by

$$
T x=x \text { and } S x=\xi \text { for all } x \in \mathfrak{V} \text { ( } \xi \text { is some element in?). }
$$

Let $\psi: \mathscr{B} \rightarrow \mathscr{B}$ defined by $\phi(a)=a-1$ for all $a \in \mathscr{B}$, where ' 1 ' is the universal element of $\mathscr{B}$.

Now there exists a sequence $\left\{x_{n}\right\}$ in $\mathfrak{V}$ defined by $x_{n}=\xi$ for all $n=1,2, \ldots$, such that

$$
\lim _{n \rightarrow \infty} S x_{n}=\lim _{n \rightarrow \infty} T x_{n}=\xi .
$$

Further, $S \mathfrak{V} \subset T \mathfrak{V}$ and

$$
\|S x-S y\|=\|0\|=\psi(M(x, y)),
$$

where $\|\cdot\|$ is any norm defined on $\mathfrak{V}$. Thus all the hypotheses of Theorem 2.2 are satisfied and $\xi$ is a common fixed point of $S$ and $T$.

\section{Acknowledgements}

The authors would like to thank the referees for their constructive comments and useful suggestions.

\section{Author details}

${ }^{1}$ Department of Mathematics, Walter Sisulu University, Mthatha 5117, South Africa ${ }^{2}$ Department of Mathematics, Pure \& Applied, Rhodes University, Grahamstown 6140, South Africa 


\section{Competing interests}

The authors declare that they have no competing interests.

Received: 16 July 2011 Accepted: 23 March 2012 Published: 23 March 2012

\section{References}

1. Ghilezan, C: Some fixed point theorems in Boolean Algebra. Publ Inst Math (Beograd). 28(42), 77-82 (1980)

2. Rudeanu, S: Boolean transformations with unique fixed points. Math Slovaca. 57, 1-10 (2007). doi:10.2478/s12175-0070010-y

3. Rao, DPRVS, Pant, R: Fixed point theorems in Boolean vector spaces. Nonlinear Anal. 74, 5383-5387 (2011). doi:10.1016/j. na. 2011.05 .021

4. Subrahmanyam, NV: Boolean vector spaces-I. Math Z. 83, 422-433 (1964). doi:10.1007/BF01111003

5. Subrahmanyam, NV: Boolean vector spaces-II. Math Z. 87, 401-419 (1965). doi:10.1007/BF01111721

6. Aamri, M, Moutawakil, DEl: Some new fixed point theorem under strict contractive conditions. J Math Anal Appl. 270, 181-188 (2002). doi:10.1016/S0022-247X(02)00059-8

7. Pant, RP: Fixed points of nonexpansive mappings and a generalized notion of compactness. Bull Cal Math Soc. 99(1), 45-52 (2007)

8. Jungck, G: Compatible mappings and common fixed points. Internat J Math Math Sci. 9(4), 771-779 (1986). doi:10.1155/ S0161171286000935

doi:10.1186/1687-1812-2012-47

Cite this article as: Mishra et al:: Fixed point theorems for a class of maps in normed Boolean vector spaces. Fixed Point Theory and Applications 2012 2012:47.

\section{Submit your manuscript to a SpringerOpen ${ }^{\odot}$} journal and benefit from:

- Convenient online submission

- Rigorous peer review

- Immediate publication on acceptance

- Open access: articles freely available online

- High visibility within the field

- Retaining the copyright to your article

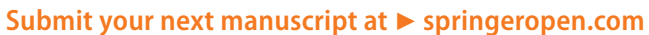

